\title{
Indicadores cientométricos normalizados: um estudo na produção científica brasileira internacional (1996 a 2011)
}

Maria Cláudia Cabrini Grácio

Docente do Programa de Pós-Graduação em Ciência da Informação da UNESP - Marília.

Ely Francina Tannuri de Oliveira

Livre-Docente em Estudos Bibliométricos em Ciência da Informação do Programa de PósGraduação e do Departamento de Ciência da Informação da UNESP - Marília.

http: / / dx.doi.org/10.1590/1981-5344/1898

Nesta pesquisa, objetiva-se: analisar comparativamente os indicadores cientométricos normalizados de produção e de citação da pesquisa brasileira, nas 27 áreas do conhecimento, a partir dos dados apresentados no portal SCImago Journal \& Country Rank, no período de 1996 a 2011; avaliar a correlação entre esses indicadores, a fim de se verificar se a inserção e o impacto apresentam-se associados no desempenho brasileiro; e agrupar as áreas por meio das similaridades dos indicadores analisados. Como procedimento, para cada área, calcularam-se a porcentagem de periódicos brasileiros, a partir da opção Journal Rankings, e o índice normalizado brasileiro para os indicadores em análise, a partir da opção Country Rankings. A seguir, calcularam-se as correlações de Pearson entre os indicadores e realizouse a análise de cluster, a fim de se agrupar as 27 áreas em função das similaridades nos indicadores. Como resultados, constatou-se que o Brasil ocupa posição acima da média dos países produtores, para todos os índices normalizados. Com exceção da média de citação, todos os demais indicadores apresentam correlações positivas. Constituíram-se quatroclusters para a atuação brasileira. Os resultados apontam que a análise conjunta e articulada dos índices normalizados possibilita uma visualização mais consistente da ciência brasileira. 


\section{International Brazilian scientific production: a scientometric study by normalized indicators (1996-2011)}

In this research, we aim at comparatively analyzing the normalized scientometric indicators of production and citation of the Brazilian research, in the 27 areas of knowledge, from the data presented in the Scimago Journal \& Country Rank gate, in the period between 1996 and 2011. Besides, we aim at assessing the correlation among these indicators in order to verify if the insertion and the impact are associated within the Brazilian performance. Eventually, we aim at grouping the areas by means of the similarities of the indicators analyzed. As our procedure, we calculated the percentage of Brazilian journals, from the Journal Rankings option, and the normalized Brazilian index for the indicators under analysis, from the Country Rankings option. Next, we calculated the Pearson correlations among the indicators and we achieved the cluster analysis, in order to group the 27 areas, according to their similarities in the indicators. As results, Brazil ranks above average among the producing countries, in all the normalized indexes. Except from the citation average, all the other indicators present positive correlations. 4 clusters were constituted for the Brazilian performance. The results show that the grouped and articulated analysis of normalized indexes enables a more consistent view of the Brazilian science

Key-words: Normalized scientometric indicators; Brazilian scientific production; Internationalization of Brazilian research.

Recebido em 14.10.2013 Aceito em 18.12.2013

\section{Introdução}

A ciência brasileira tem crescido de modo significativo nas últimas décadas, com consequente aumento da participação do país na produção científica e no conhecimento mainstream, que pode ser visualizado por meio dos dados das bases internacionais (LEITE; MUGNAINI; LETA, 2011). 
Nesse contexto, a taxa de crescimento da produção científica do Brasil, de forma geral, superou a média internacional, com um aumento de seis vezes nas duas últimas décadas do século $X X$, uma vez que no ano de 2002 ocupava a $17^{a}$ posição no ranking de produção científica (ZANOTTO, 2002) e em 2011 passou a ocupar a 13a posição (SCImago, 2012).

O aumento expressivo da produção científica brasileira proporcionou maior inserção do país em âmbito internacional, passando a ser visível e necessitando de metodologias para mostrar, de forma comparativa, o desempenho brasileiro nas diferentes áreas do conhecimento.

Dentre os métodos para a análise e avaliação da produção científica, destacam-se os estudos cientométricos, que, por meio de seus indicadores absolutos e normalizados (dentre eles, os de produção e citação), contribuem para a visualização do comportamento da ciência nas diferentes áreas do conhecimento. Os indicadores normalizados identificam, de forma comparativa, questões relativas à produção e ao impacto científico atinentes às diferentes áreas do conhecimento, na medida em que padronizam as unidades de medida (GLÄNZEL et al, 2009).

Considerando a problemática apresentada, esta pesquisa objetiva, de forma geral, realizar um estudo da contribuição dos indicadores normalizados para os estudos cientométricos que buscam visualizar, de forma comparativa, o desenvolvimento científico de diferentes áreas do conhecimento, destacando a presença brasileira nas 27 áreas do conhecimento apresentadas pelo Portal SCImago Journal \& Country Rank, no período de 1996 a 2011.

De forma mais específica, propõe-se a analisar a porcentagem relativa das revistas brasileiras indexadas internacionalmente e os indicadores cientométricos normalizados de produção e de citação da pesquisa brasileira, nas 27 áreas do conhecimento, a partir dos dados apresentados no SCImagoJR. Ainda, avaliar a correlação entre os seguintes indicadores: porcentagem de revistas brasileiras indexadas na base, índice normalizado de produção, de total de citações, de citações por documento, de autocitação e do índice h, a fim de se verificar se a inserção e impacto apresentam-se associados no desempenho brasileiro nas áreas avaliadas. Por último, agrupar as áreas do conhecimento por meio das similaridades dos índices normalizados cientométricos e da porcentagem de revistas brasileiras indexadas na baseSCImagoJr.

Este trabalho se justifica em razão da escassez de estudos existentes, em especial no Brasil, referentes aos indicadores normalizados, e pela contribuição, de forma analítica, para o desenvolvimento da temática em estudo, ao oferecer subsídios teóricos e metodológicos, fortalecendo sua compreensão e uso, bem como pela necessidade de estudos eminentemente brasileiros sobre o tema. Justifica-se a escolha da ciência brasileira mainstream como universo de aplicação dos indicadores normalizados por contribuir para a visualização, de forma comparativa, do desenvolvimento das várias áreas científicas do país, identificando aquelas que se destacam em produção e impacto. 


\section{Indicadores cientométricos absolutos e normalizados de produção e citação}

Em decorrência dos aspectos apontados, a ciência brasileira tem crescido de forma expressiva, particularmente nos últimos 25 anos (ZANOTTO, 2002; LETA; CHAIMOVICH, 2002; LETA; GLÄNZEL; THIJS, 2006; LEITE; MUGNAINI; LETA, 2011). Neste período, o desenvolvimento científico levou o Brasil à posição de liderança no contexto da comunidade científica latino-americana, com intensa colaboração científica internacional, tanto regional como com a América do Norte e Europa (GLÄNZEL; LETA; THIJS, 2006).

Nesse contexto, os estudos bibliométricos constituem uma abordagem objetiva que, associada às análises contextuais, contribui para a avaliação da produção científica e tecnológica de uma área, de um grupo de instituições ou países.

A análise da produção científica envolve um grupo de indicadores bibliométricos, absolutos e relativos ou normalizados, que podem ser agrupados em indicadores de produção, citação e ligação (OKUBO, 1997; SPINAK, 1998; NARIN et al, 1994; CALLON et al., 1993). Entre os diversos aspectos analisados, esses indicadores evidenciam os esquisadores, as temáticas, as instituições, as áreas do conhecimento e os países mais produtivos e de maior impacto, assim como as linhas de pesquisa de uma área do conhecimento, as redes de colaboração científica, de citação e cocitação entre pesquisadores, instituições ou países. Nesta pesquisa, enfocam-se os indicadores de produção e de citação.

O indicador absoluto de produção é constituído pelo número de publicações de um autor, instituição ou país, com o objetivo de evidenciar sua inserção junto à comunidade científica a qual pertence, dando visibilidade àqueles mais produtivos e às temáticas mais relevantes de uma área do conhecimento (OLIVEIRA; GRÁCIO, 2009).

Os indicadores absolutos de citação permitem identificar a visibilidade e o impacto de um pesquisador ou país dentro de uma comunidade científica. Entre eles, destacam-se: o total de citações, o número de citações por documento, a autocitação e o índice $\mathrm{h}$ de um pesquisador ou país.

O total de citações é considerado indicador simples, expresso por uma quantidade absoluta, independente da grandeza do universo estudado. O número de citações por documento, calculado pela média de citações, é um indicador que contextualiza o impacto de um pesquisador, instituição ou país ao relativizar as citações em função do número de documentos produzidos. Os indicadores de autocitação têm por objetivo avaliar o comportamento auto-referente dos pesquisadores, instituições ou países. Glänzel (2003) argumenta que as autocitações constituem parte essencial da comunicação científica e que, em geral, não há razão para condená-las. Indicadores baseados em autocitações são medidas 
complementares valiosas aos indicadores clássicos (total de citações e citações por documento) para a avaliação bibliométrica.

Ainda entre os indicadores de citação, Hirsch (2005) propôs o índice h para caracterizar a produção científica acumulada de um pesquisador, instituição ou país, e o define como: "um cientista tem índice $\mathrm{h}$ se $\mathrm{h}$ de seus Np artigos tem pelo menos $h$ citações cada e os outros (Np-h) artigos tem menos que h citações cada" (HIRSCH, 2005, p. 1), em que Np é 0 total de artigos publicados. $O$ índice $h$ está associado com a distribuição das citações dos documentos, e o valor depende da natureza da área de pesquisa. Pesquisadores e países vindos de áreas que não estão na ciênciamainstream não obterão valores altos de índice $h$, mesmo se trabalharem em áreas mais significativas da ciência (OLIVEIRA; GRÁCIO, 2011).

Hirsch (2005) destaca que um único valor nunca será suficiente para descrever amplamente o perfil multifacetado de um pesquisador, instituição ou país, tornando necessária a combinação de vários indicadores em sua avaliação.

Apesar da importância dos indicadores absolutos de produção e citação para análise e compreensão da contribuição, inserção e impacto de um pesquisador ou país dentro de uma área do conhecimento, esses indicadores têm diversas limitações quando usados para comparar o desempenho entre diferentes áreas do conhecimento. Essa questão decorre das especificidades e peculiaridades das áreas, uma vez que as disciplinas científicas diferem fortemente na prática de citação, tendo como consequência o fato de o número típico de citações, que um artigo em uma dada área recebe, poder diferir fortemente do número de citações típico de outra área.

Consequentemente, os indicadores absolutos levam a procedimentos de avaliação injustos quando se realizam análises comparativas das áreas, podendo resultar em análises distorcidas da inserção e impacto das diferentes áreas do conhecimento. Nessa direção, Persson, Glänzel e Danell (2004) apontam que o uso indiscriminado desses indicadores pode levar muitas vezes a conclusões equivocadas e recomendam o uso de medidas de normalização e indicadores relativos a fim de fortalecer a validade das conclusões. Isso posto, há uma crescente tendência no sentido de formular e debater procedimentos de normalização, que possibilitem eliminar a dependência do contexto da área e, assim, oferecer critérios mais fidedignos na comparação entre disciplinas, áreas, instituições ou em nível macro de agrupamento, uma vez que padronizam as unidades de medida (GLÄNZEL et al, 2009, LI; RADICCHI; CASTELLANO; RUIZ-CASTILHO, 2013).

Para superar as questões apontadas, dentre os procedimentos de normalização propostos, Li, Radicchi, Castellano e Ruiz-Castilho (2013) destacam a normalização pela média da área, pela mediana da área, pelo método de normalização de dois parâmetros reversos, normalização de Glänzel e pela taxa de permuta. Os autores destacam, entre os procedimentos que conduzem a bons resultados, a normalização pela média da área. Autores como Moed (2009) e Herranz e Ruiz-Castillo 
(2012) trabalham com formulações de índices normalizados em que há combinações de diferentes categorias, seja de tema, de área ou tipo de documento.

Para estudos comparativos entre áreas, um indicador normalizado pela média pode ser definido como o quociente entre o valor do indicador obtido para o indivíduo (seja uma instituição ou país) dividido pela média da área (MOED, 2009). Como resultado, esse indicador padroniza o comportamento de um indivíduo, de forma a situá-lo em relação à tendência global (média) observada na área. Nesta pesquisa, os indivíduos se referem aos países.

O indicador normalizado é calculado pela fórmula:

em que:

$\mathrm{IN}_{\mathrm{j}}=$ índice normalizado para o país $\mathrm{j}$;

$\mathrm{I}_{\mathrm{j}}=$ valor do indicador absoluto para o país $\mathrm{j}$;

$\mathrm{MI}_{\mathrm{g}}=$ média do indicador da área em estudo, calculada por: em que:

$\mathrm{n}$ = quantidade de países que publicaram na área em estudo;

$\mathrm{I}_{\mathrm{i}}=$ valor do indicador para o país $\mathrm{i}, \operatorname{com} 1 \leq \mathrm{i} \leq \mathrm{n}$.

O valor 1 (um) obtido para o índice normalizado (IN) indica que, em relação ao indicador em estudo, o desempenho do país (nesta pesquisa, Brasil) é igual ao comportamento médio do grupo (nesta pesquisa, países) de produtores na área. Ainda, valores abaixo de 1 significam que o país está abaixo do que é esperado para o grupo de países, na área em estudo; valores acima de 1 apontam que o país apresenta um desempenho acima do comportamento médio do grupo.

\section{Procedimentos metodológicos}

Inicialmente, em novembro de 2012, os dados foram levantados no portal SCImago Journal \& Country Rankings, por meio dos seguintes procedimentos: na opção Journal Rankings, para cada uma das 27 áreas do conhecimento, tomou-se o total de periódicos existentes e de periódicos brasileiros. A partir destes dados, calculou-se o percentual de periódicos do Brasil em relação ao total indexado na área, apresentados na primeira coluna de valores (\%) na Tabela 1.

Em seguida, ainda no portal SCImago Journal \& Country Rankings, na opção Country Rankings, para cada uma das 27 áreas, recuperou-se a planilha Excel com o conjunto de países produtores, com os seguintes indicadores: documentos citáveis, total de citações, autocitações, citações por documentos e índice h. Para cada uma das 27 áreas, calcularam-se as médias dos indicadores em análise, relativa aos países. Destaque-se que "documentos citáveis" está sendo utilizado, nesta pesquisa, como "total de documentos" produzidos. 
A seguir, para cada área, calculou-se o índice normalizado do Brasil para cada indicador arrolado acima, em relação às médias obtidas, apresentados na Tabela 1.

Para cada coluna de índice normalizado, presente na Tabela 1 , calcularam-se média, desvio padrão, mínimo, máximo e coeficiente de variação, em relação ao conjunto de 27 áreas.

Em continuidade, calcularam-se as correlações de Pearson entre os seguintes indicadores: porcentagem de periódicos brasileiros, índice normalizado de total de documentos, de total de citações, de autocitações, de citações por documento e de índice h.

Por fim, realizou-se a análise de cluster hierárquico, pelo método Ward, com medida de similaridade de Distância Euclidiana Quadrática e dados normalizados pelas variáveis, a fim de se agrupar as 27 áreas analisadas, em função de suas semelhanças em relação aos indicadores normalizados, considerados de forma conjunta e simultânea. A visualização dos clusters foi feita por meio do dendograma.

A fim de estabelecer o perfil e as características dos grupos constituídos, a partir da análise de clusters, foram calculadas as médias, desvios padrão e coeficientes de variação, para cada indicador, por grupo, e realizadas as análises comparativas intergrupos.

\section{Apresentação e análises de dados}

A Tabela 1 apresenta o desempenho da ciência brasileira nas diferentes áreas do conhecimento presentes no portal SCImagoJR. Inicialmente, destaca-se que, para todos os índices normalizados, a média para as 27 áreas se encontra acima de 1 , indicando que, em média, em todos esses índices, o Brasil ocupa posição acima do comportamento médio do grupo de países produtores. Observase ainda, que, para o índice normalizado do total de documentos, em todas as 27 áreas, o Brasil se encontra acima da média, isto é, apresenta índice normalizado de total de documentos produzidos acima de 1 . Complemente-se que o índice $\mathrm{h}$ normalizado é aquele indicador com menor variação no desempenho brasileiro. Por outro lado, o índice normalizado de autocitação é aquele com maior variação no desempenho brasileiro nas 27 áreas, confirmando a pesquisa de Glänzel e Thijs (2004), segundo a qual a autocitação varia muito entre as áreas do conhecimento.

Quanto à porcentagem de periódicos brasileiros indexados na Scopus, observa-se que, em média, a participação brasileira é de 1,5\% do total de periódicos indexados. As áreas de Odontologia, Veterinária e "Agricultura e Ciências Biológicas" são aquelas que, nesta ordem, apresentam maior participação de periódicos brasileiros. Por outro lado, as áreas de Energia, "Ciência da Computação" e Matemática apresentam as menores porcentagens de periódicos brasileiros indexados. Assim, há áreas da ciência brasileira que se diferenciam das demais, algumas por uma presença mais significativa de periódicos brasileiros e outras de menor inserção. 
Em relação ao índice normalizado de total de documentos, em média, a produção científica brasileira está 3,2 vezes acima da média dos países produtores. Destaca-se que as áreas de Veterinária, "Agricultura e Ciências Biológicas" e "Imunologia e Microbiologia" são aquelas que apresentam os maiores índices normalizados, com a primeira delas apresentando total de documentos produzidos 10,4 vezes acima da média dos países produtores. Por outro lado, as áreas "Negócios, Gestão e Contabilidade", "Economia, Econometria e Finanças" e Multidisciplinar são aquelas com menores índices normalizados de total de documentos produzidos, embora também estejam acima da média (acima de 1) para as

respectivas

áreas.

Tabela 1 - Percentual de periódicos brasileiros e índices normalizados de produção e de citação do Brasil nas 27 áreas

\begin{tabular}{|c|c|c|c|c|c|c|}
\hline Área & $\%$ & $\begin{array}{l}\text { Total } \\
\text { documentos* }\end{array}$ & $\mid \begin{array}{l}\text { Total } \\
\text { citações* }\end{array}$ & Autocitações* & $\begin{array}{l}\text { Citações } \\
\text { por doc.* }\end{array}$ & indice \\
\hline Agricultura e Ciências Biológicas & 3.5 & 7.6 & 3.7 & 5.2 & 0.9 & 2.9 \\
\hline Artes and Humanidades & 0.8 & 2.1 & 1.6 & 0.6 & 1.1 & 2 \\
\hline $\begin{array}{llll}\begin{array}{l}\text { Bioquímica, } \\
\text { Molecular }\end{array} & \text { Genética } & \text { e } & \text { Biol. } \\
\end{array}$ & 0.8 & 2.9 & 1.4 & 1.4 & 1 & 2.5 \\
\hline Ciência da Computação & 0.2 & 2.1 & 1.2 & 1.2 & 0.9 & 2.6 \\
\hline Ciências Ambientais & 1.8 & 3.4 & 2.5 & 2.6 & 1.3 & 3.1 \\
\hline Ciências da Decisão & 0.6 & 2 & 1.5 & 1.6 & 1.3 & 2.3 \\
\hline Ciências da Saúde & 2.0 & 2.6 & 0.8 & 0.7 & 1.2 & 2.1 \\
\hline Ciências dos Materiais & 1.3 & 2.3 & 1.7 & 1.6 & 1.1 & 2.3 \\
\hline Ciências Planetárias e da Terra & 1.5 & 2.6 & 1.8 & 1.5 & 1.1 & 2.7 \\
\hline Ciências Sociais & 1.8 & 2.9 & 0.9 & 0.8 & 1.2 & 2.3 \\
\hline Economia, Econometria e Finanças & 1.2 & 1.5 & 0.6 & 0.3 & 1.9 & 2 \\
\hline Energia & 0.0 & 2.3 & 1.9 & 1.8 & 0.9 & 2.5 \\
\hline Enfermagem & 1.9 & 4.3 & 1.2 & 1.2 & 1.9 & 2.9 \\
\hline Engenharia & 0.8 & 1.9 & 1.5 & 1.2 & 1 & 2.8 \\
\hline Engenharia Química & 1.2 & 2.8 & 2.5 & 2.5 & 1.2 & 2.7 \\
\hline Farmacologia, Toxic. e Farmácia & 0.9 & 3.8 & 2.5 & 3.3 & 1.1 & 2.7 \\
\hline Física e Astronomia & 0.4 & 2.8 & 2 & 1.8 & 1.1 & 2.8 \\
\hline Imunologia e Microbiologia & 1.1 & 4.9 & 2.7 & 3.1 & 1 & 2.6 \\
\hline Matemática & 0.3 & 2.9 & 2.4 & 2.6 & 1.3 & 2.7 \\
\hline Medicina & 1.3 & 3.9 & 1.8 & 1.7 & 0.9 & 2.8 \\
\hline Multidisciplinar & 1.4 & 1.8 & 0.7 & 0.4 & 0.7 & 2.5 \\
\hline Negócio, Gestão e Contabilidade & 0.5 & 1.2 & 0.5 & 0.3 & 1.5 & 1.9 \\
\hline Neurociência & 1.6 & 3.5 & 1.5 & 1.5 & 0.4 & 2.9 \\
\hline Odontologia & 5.7 & 2.3 & 7.6 & 12 & 1.4 & 3.9 \\
\hline Psicologia & 2.5 & 2.3 & 0.6 & 0.4 & 1.3 & 2.5 \\
\hline Química & 1.1 & 2.8 & 2 & 2.3 & 1.1 & 2.6 \\
\hline Veterinária & 4.7 & 10.4 & 5 & 8.4 & 0.9 & 3 \\
\hline Média & 1.5 & 3.2 & 2.0 & 2.3 & 1.1 & 2.6 \\
\hline Desvio padrão & 1.3 & 1.9 & 1.5 & 2.6 & 0.3 & 0.4 \\
\hline Coeficiente de variação & $37 \%$ & $25 \%$ & $40 \%$ & $49 \%$ & $35 \%$ & $14 \%$ \\
\hline Mínimo & 0 & 1.2 & 0.5 & 0.3 & 0.4 & 1.9 \\
\hline Máximo & 5.7 & 10.4 & 7.6 & 12 & 1.9 & 3.9 \\
\hline
\end{tabular}

*índice normalizado

Fonte: elaboração dos autores, a partir dos dados do SCImagoJR em novembro de 2012. 
Em relação ao índice normalizado do total de citações recebidas, a média obtida para as 27 áreas está duas vezes acima da média das respectivas áreas, com destaque para Odontologia, Veterinária e "Agricultura e Ciências Biológicas", em que os índices normalizados de total de citação são os maiores observados para a ciência brasileira. Tal tendência está alinhada com as maiores porcentagens de periódicos brasileiros nas respectivas áreas. Para as áreas de Veterinária e "Agricultura e Ciências Biológicas", os altos índices normalizados de total de citação estão também alinhados com os altos índices normalizados de documentos produzidos. Por outro lado, as áreas "Negócios, Gestão e Contabilidade", "Economia, Econometria e Finanças" e Psicologia são aquelas com os menores índices normalizados de total de citação, com valores menores que 1 , indicando que o impacto das citações está abaixo da média dos países participantes destas áreas.

Quanto ao índice normalizado de autocitação, obteve-se a média de 2,3 para o conjunto das áreas, com as áreas de Odontologia, Veterinária e "Agricultura e Ciências Biológicas" apresentando os maiores índices normalizados para esse indicador. As duas últimas áreas também estão contempladas com as maiores porcentagens de periódicos brasileiros na ciência mainstream e os maiores índices normalizados de total de documentos e de total de citações, sugerindo que nestas áreas o Brasil já constitui referência científica. Entre as hipóteses para esse desempenho, apontam-se a consolidação e a tradição das pesquisas nestas áreas (LETA; GLÄNZEL; THJIS, 2006), favorecidas pela característica geográfica do país, que potencializa as possibilidades de pesquisa. Neste contexto, destaca-se o papel fundamental da Empresa Brasileira de Pesquisa Agropecuária (EMBRAPA) no crescimento da produção científica agrícola brasileira (LANDIM, 2010). Ainda, destaca-se a interdisciplinaridade entre as duas áreas Veterinária e "Agricultura e Ciências Biológicas", produzindo pesquisas de interesse comum.

Por outro lado, com os menores índices normalizados de autocitação, aparecem as áreas de "Negócio, Gestão e Contabilidade", "Economia, Econometria e Finanças" e Psicologia, compatibilizando-se com o índice normalizado de total de citações. Para as duas primeiras áreas, a posição de menor índice é alinhada com o índice normalizado de total de documentos produzidos.

Em relação ao índice normalizado de citações por documento, obteve-se a média 1,1, valor este muito próximo da média das respectivas áreas. Além disso, esta média é a menor obtida entre indicadores analisados para o Brasil, alinhando-se com os resultados encontrados por Glänzel, Leta e Thijs (2006), que apontam uma visibilidade da pesquisa brasileira mais baixa que a média e um impacto relativamente baixo da citação, acompanhado por altos valores de autocitação. Destaca-se que as áreas de "Economia, Econometria e Finanças", Enfermagem e "Negócio, Gestão e Contabilidade" apresentam os maiores índices normalizados de citação por documento, entre as 27 áreas analisadas. Observa-se, porém, que as áreas "Economia, Econometria e Finanças" e "Negócio, Gestão e Contabilidade" foram 
destacadas como aquelas que apresentaram os menores valores para os indicadores normalizados já analisados, a saber: total de documentos produzidos, total de citações recebidas e autocitações, sugerindo que, embora essas áreas não se destaquem em volume de produção, seu impacto, refletido pelas citações por documento junto à comunidade científica internacional, é significativo, dada a baixa autocitação. Por outro lado, as áreas de Neurociências e Multidisciplinar apresentam-se com os menores índices normalizados de citações por documento.

Entre todos os indicadores normalizados, o índice h normalizado é aquele que se apresenta mais homogêneo entre as 27 áreas analisadas, com média igual a 2,6 vezes acima da média global, das respectivas áreas. Destacam-se as áreas de Odontologia, "Ciências Ambientais" e Veterinária com os maiores índices normalizados; por outro lado, as áreas de "Negócio, Gestão e Contabilidade", "Economia, Econometria e Finanças" e "Artes e Humanidades" apresentam-se com os menores índices. As áreas de "Negócio, Gestão e Contabilidade", "Economia, Econometria e Finanças" estão em concordância com a afirmação de Glänzel (2006) e Vieira e Gomes (2010), de que o índice h depende do número de documentos publicados, uma vez que são aquelas com os menores índices normalizados de produção e de índice h. Todavia, essa afirmação não se ratifica nas áreas de Odontologia e "Ciências ambientais", com altos índices h normalizados e volume de produção não destacado, em relação às demais áreas.

A fim de se verificar se a inserção e o impacto da ciência brasileira apresentam-se associados nas 27 áreas, calculou-se a correlação de Pearson entre os índices analisados, a saber: porcentagem de revistas brasileiras indexadas na Scopus, índice normalizado de produção, de total de citações, de autocitações, de citações por documento e de índice h. Os resultados são apresentados na Tabela 2 .

A partir da análise da Tabela 2, observa-se que, com exceção do índice normalizado de citações por documento, todos os demais indicadores em estudo apresentam correlações positivas estatisticamente significantes entre si, com maior ou menor intensidade. Destacam-se as correlações mais fortes entre o índice normalizado de total de citação e de autocitações $(0,99 * *)$ e entre índice normalizado de autocitações e porcentagem de periódicos brasileiros indexados na área $\left(0,81^{* *}\right)$, significando que o total de citações está fortemente associado e dependente da autocitações no país, nas áreas em estudo, e este último cresce à medida que cresce a porcentagem de periódicos brasileiros indexados nas diferentes áreas. Tabela 2 - Valores da correlação de Pearson entre porcentagem de periódicos brasileiros (\%) e os índices normalizados de produção e citação

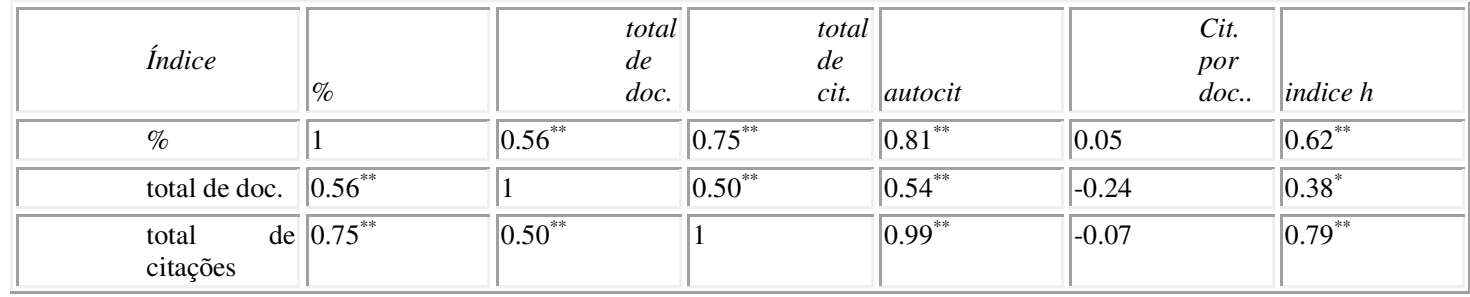




\begin{tabular}{|l|l|l|l|l|l|l|}
\hline autocitações & $0.81^{* *}$ & $0.54^{* *}$ & $0.99^{* *}$ & 1 & -0.05 & $0.77^{* *}$ \\
\hline Cit. por doc. & 0.05 & -0.24 & -0.07 & -0.05 & 1 & -0.16 \\
\hline índice h & $0.62^{* *}$ & $0.38^{*}$ & $0.79^{* *}$ & $0.77^{* *}$ & -0.16 & 1 \\
\hline
\end{tabular}

* significante a $\square=0.05$. ** significante $a=0.01$ Fonte:Elaborado pelas autoras

Em relação ao índice normalizado de citações por documento, a ausência de correlação com os demais índices sugere que os valores de citações por documento não estão associados ao aumento da porcentagem de periódicos brasileiros e nem ao incremento da produção brasileira nas áreas do conhecimento. Assim, áreas com menor participação brasileira na produção científica e/ou com menor inserção de periódicos brasileiros podem apresentar um maior impacto, por documento, na ciênciamainstream, como acontece com as áreas de "Negócio, Gestão e Contabilidade" e "Economia, Econometria e Finanças". Contrapondo-se a esse comportamento, as áreas de Veterinária e "Agricultura e Ciências Biológicas" apresentam altos índices de porcentagem de periódicos indexados e de total de documentos, porém o índice normalizado de citações por documento apresenta-se abaixo da média global dos países produtores.

Apresenta-se, na Figura 1, o cluster das 27 áreas apresentadas na base Scopus, agrupadas segundo as similaridades dos índices da ciência brasileira, em que se destacam 4 grupos, a saber: o primeiro grupo, constituído por 14 áreas, iniciando-se com Química até Neurociências; o segundo grupo, constituído por 10 áreas, iniciando-se em Ciências da Saúde até Enfermagem; o terceiro grupo, composto por 2 áreas "Agricultura e Ciências Biológicas" e Veterinária; e o último grupo, constituído por uma única área - Odontologia.

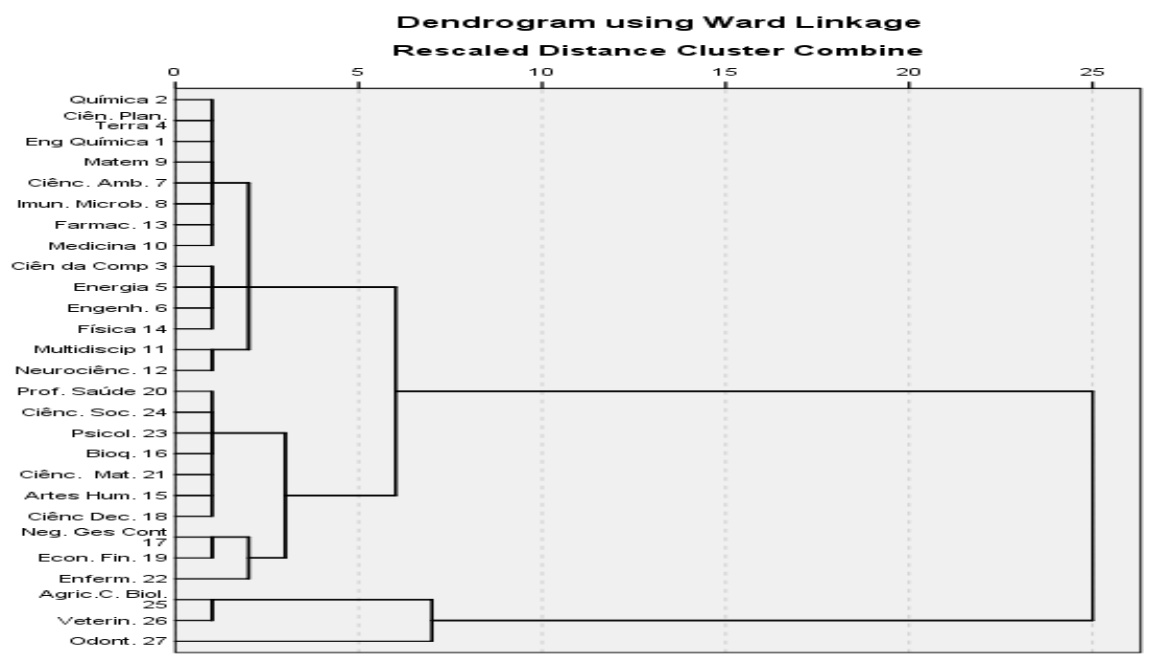

Figura 1 - Cluster das 27 áreas segundo similaridades nos indicadores analisados 
Apresenta-se a Tabela 3 com os valores de média, desvio padrão e coeficiente de variação para os índices normalizados utilizados na análise de cluster, para os quatro grupos constituídos.

O primeiro grupo, composto pela maioria (14) das 27 áreas em estudo, apresenta em relação à porcentagem de periódicos indexados a menor média entre os grupos constituídos, embora seja o indicador com maior dispersão dentro deste grupo, representado pelo alto valor do coeficiente de variação. Em relação a todos os índices normalizados, observa-se que as respectivas médias não se apresentam destacadas em relação às médias dos outros três grupos, seja por altos ou baixos valores médios normalizados. Apesar de ser o maior agrupamento de áreas, com exceção da autocitação, os demais índices normalizados apresentam-se com baixa variabilidade dentro do grupo, explicitado pelos coeficientes de variação abaixo de 30\%.

O grupo 2, apesar de apresentar um volume de produção menos significativo, bem como os periódicos brasileiros pouco inseridos na ciência internacional, tem impacto destacado em relação aos outros três grupos, dada a alta média dos índices normalizados de citações por documento, porém com baixo comportamento auto-referente. Complemente-se que neste grupo o índice normalizado de autocitação, porcentagem de periódicos indexados e de total de citações são aqueles com maior variação no grupo. Por outro lado, o índice $\mathrm{h}$ normalizado apresenta-se como 0 mais homogêneo dentro do grupo Tabela 3 - Estatísticas descritivas da porcentagem de periódicos brasileiros e indicadores normalizados para os quatro agrupamentos

\begin{tabular}{|c|c|c|c|c|c|c|c|}
\hline \multirow[t]{3}{*}{ Grupo } & Estatísticas descritivas & $\%$ & $\begin{array}{l}\text { total } \\
\text { documentos }\end{array}$ & $\begin{array}{l}\text { total } \\
\text { citações }\end{array}$ & autocit & Cit. por doc. & indic \\
\hline & Média & 1.0 & 3.0 & 1.9 & 2.0 & 1.0 & 2.7 \\
\hline & Desvio Padrão & 0.6 & 0.9 & 0.6 & 0.8 & 0.2 & 0.2 \\
\hline \multicolumn{8}{|l|}{ Grupo 1} \\
\hline & Coef. de variação & $57 \%$ & $29 \%$ & $30 \%$ & $41 \%$ & $24 \%$ & $6 \%$ \\
\hline & Média & 1.3 & 2.4 & 1.1 & 0.9 & 1.4 & 2.3 \\
\hline & Desvio Padrão & 0.7 & 0.9 & 0.5 & 0.5 & 0.3 & 0.3 \\
\hline \multicolumn{8}{|l|}{ Grupo 2} \\
\hline & Coef. de variação & $50 \%$ & $36 \%$ & $42 \%$ & $58 \%$ & $24 \%$ & $13 \%$ \\
\hline & Média & 4.1 & 9.0 & 4.4 & 6.8 & 0.9 & 3.0 \\
\hline & Desvio Padrão & 0.9 & 2.0 & 0.9 & 2.3 & 0.0 & 0.1 \\
\hline \multicolumn{8}{|l|}{ Grupo 3} \\
\hline & Coef. de variação & $21 \%$ & $22 \%$ & $21 \%$ & $33 \%$ & $0 \%$ & $2 \%$ \\
\hline Grupo 4 & Média & 5.7 & 2.3 & 7.6 & 12.0 & 1.4 & 3.9 \\
\hline
\end{tabular}

O terceiro grupo, composto pelas áreas de "Agricultura e Ciências Biológicas" e Veterinária, apresenta altos valores em cinco dos seis índices analisados, a saber: $2^{a}$ e $3^{a}$ maiores porcentagens de periódicos indexados na base Scopus, os maiores índices normalizados de total de documentos produzidos, bem como os $2^{\circ}$ e $3^{\circ}$ mais altos índices normalizados de total de citação, de autocitações e de índice $h$. Por outro lado, esse grupo é aquele que apresenta a menor média de índice normalizado de citações por documento.

Como mencionado, o último grupo é constituído por uma única área, Odontologia. Dentre as 27 áreas analisadas, esta se destaca das demais por apresentar valores bastante acima daqueles observados para as 
outras áreas em quatro dos seis índices analisados, a saber: maior porcentagem de periódicos indexados e os mais altos índices normalizados de total de citação, de autocitação e de índice h. Destaque-se que esses índices estão alinhados com o estudo de Grácio et al. (2012), o qual aponta que o impacto e a visibilidade da Odontologia do Brasil na ciência internacional decorrem do maior acesso às bases de dados internacionais, bem como a internacionalização dos programas de pós-graduação brasileiros, entre outras razões.

Com exceção do índice normalizado de documentos produzidos, os grupos 3 e 4 apresentam índices com valores aproximados, visualizados pela sua proximidade de posição no dendograma, apresentado na Figura 3

\section{Considerações Finais}

Considerando que esta pesquisa se propôs a analisar, de forma comparativa, os indicadores cientométricos normalizados de produção e de citação da pesquisa brasileira, nas 27 áreas do conhecimento apresentadas pelo SCImagoJR, destaca-se que o Brasil ocupa posição acima da média do grupo de países produtores, para todos os índices normalizados em análise.

Em relação à porcentagem de periódicos brasileiros indexados na base Scopus, destacam-se as áreas de Odontologia, Veterinária, "Agricultura e Ciências Biológicas", com as presenças mais significativas na ciência mainstream.

Observa-se que para o índice normalizado do total de documentos, em todas as 27 áreas, o Brasil apresenta produção acima da média global das respectivas áreas. Quanto ao índice normalizado do total de citações recebidas, destacam-se as áreas de Odontologia, Veterinária e "Agricultura e Ciências Biológicas", em que os valores são os maiores observados para a ciência brasileira. O índice normalizado de autocitação é o indicador com a maior variação no comportamento brasileiro nas 27 áreas. O índice normalizado de citações por documento é o indicador com menores valores observados nas diferentes áreas, com muitas áreas apresentando valores normalizados abaixo da média do conjunto de países produtores. Além disso, este índice apresentou a menor média entre os índices analisados para o Brasil. Complete-se, ainda, que o índice $\mathrm{h}$ normalizado é aquele indicador com menor variação no desempenho brasileiro.

Com exceção do índice normalizado de citações por documento, todos os demais indicadores em estudo apresentam correlações positivas estatisticamente significantes entre si, com maior ou menor intensidade.

A atuação brasileira nas 27 áreas do conhecimento foi agrupada segundo as similaridades dos índices analisados, destacando-se 4 grupos. O primeiro grupo, composto pela maioria das áreas em estudo, apresenta tendência de baixa porcentagem de periódicos brasileiros indexados e todos os índices normalizados sem destaque. O grupo 2, apesar de apresentar um volume de produção menos significativo, bem como os periódicos brasileiros pouco inseridos na ciência internacional, tem 
impacto significativo em suas respectivas áreas. O terceiro grupo, composto pelas áreas de "Agricultura e Ciências Biológicas" e Veterinária, com exceção do índice normalizado de citação por documento, apresenta altos valores nos indicadores analisados. O último grupo, constituído unicamente pela área de Odontologia, se destaca por apresentar os maiores índices normalizados em relação às 27 áreas, com exceção do índice normalizado de total de documentos e de citação por documento.

Assim, com exceção do índice normalizado de documentos produzidos, as áreas de "Agricultura e Ciências Biológicas", Veterinária e Odontologia, representadas pelos grupos 3 e 4, apresentam índices com valores aproximados.

Em síntese, analisaram-se os índices normalizados que descrevem a produção e o impacto da ciência brasileira, de forma comparativa entre as 27 áreas do conhecimento. Considerando que o Brasil apresentou desempenho diferente nestes índices, nas diferentes áreas, aponta-se a necessidade da análise conjunta e articulada de diferentes índices normalizados a fim de se descrever diferentes características da ciência brasileira e possibilitar uma visualização mais ampla e consistente do universo estudado.

Finalizando, dadas as limitações dos indicadores absolutos de produção e de citação para análise comparativa da inserção e do impacto das diferentes áreas do conhecimento de um país, pelas suas especificidades, decorrentes das práticas de produção e de citação, esta pesquisa contribuiu para a compreensão dos indicadores normalizados, que possibilitam a comparação entre as diversas áreas, ao padronizarem as unidades de medida, eliminando a dependência do contexto em que o conhecimento foi produzido

\section{Referências}

CALLON, M.; COURTIAL, J. P.; PENAN, H. Cienciometría: la medición de la atividad científica: de la bibliometría a la vigilancia tecnológica. Astúrias: Trea, 1995.

GLÄNZEL, W. Bibliometrics as a research field: a course on theory and application of bibliometric indicators. Belgium. 2003. Available from: http://citeseerx.ist.psu.edu/viewdoc/download?doi=10.1.1.97.5311\&rep=rep1\& type $=$ pdff. Accesss in: Nov. $8^{\text {th }} 2010$.

Glänzel, W. On the h-index - A mathematical approach to a new measure of publication activity and citation impact. Scientometrics, Budapeste, v. 67, n.2, p.315-321, 2006.

GLÄNZEL, W.; THIJS, B. The influence of self-citations on bibliomterics macro citations.Scientometrics, Budapeste, v. 59, p. 281-310, 2004.

GLÄNZEL, W.; LETA, J.; THIJS, B. Science in Brazil. Part 1: A macro-level comparative study. Scientometrics, Budapeste, v. 67, n. 1, p. 67-86, 2006. 
GLÄNZEL, W.; THIJS, B.; SCHUBERT, A.; DEBACKERE, K. Subfield-specific normalized relative indicators and a new generation of relational charts: Methodological foundations illustrated on the assessment of institutional research performance.Scientometrics, Budapeste, v. 78, n. 1, p. 165-188, 2009.

GRÁCIO, M.C.C.; OLIVEIRA, E. F. T.; GURGEL, J. A.; ESCALONA, M. I.; GUERRERO, A. P. Dentistry scientometric analysis: a comparative study between Brazil and other most productive countries in the area. Scientometrics, Budapeste, v. 92, p. 1-17, 2012.

Herranz, N. \& Ruiz-Castillo, J. Sub-field normalization in the multiplicative case: Average-based citation indicators. Journal of Informetrics, Amsterdam, v. 6, p.543-556, 2012.

HIRSCH. J. E. An index to quantify an individual's scientific research output. ArXiv:physics/0508025v5 [physics.soc-ph. September 2005. Available from: < http://arxiv.org/abs/physics/0508025. Access in: Apr. 18th 2009.

LANDIM, R. Brasil já é o terceiro maior exportador agrícola do mundo. $O$ Estado de S. Paulo, São Paulo, 2010. Disponível em: http://www.estadao.com.br/noticias/ economia,brasil-ja-e-o-terceiro-maiorexportador-agricola-do-mundo,520500,0.htm . Acesso em: 14 dez. 2012.

LEITE, P.; MUGNAINI, R.; LETA, J. A new indicator for international visibility: exploring Brazilian scientific community. Scientometrics, Budapeste, v. 88, p. 11-319, 2011.

LETA, J.; CHAIMOVICH, H. (Recognition and international collaboration: the Brazilian case. Scientometrics, Budapeste, v.53, n. 3, p. 325-335, 2002.

LETA, J.; GLÄNZEL, W.; THIJS, B. Science in Brazil. Part 2: Sectoral and institutional research profiles. Scientometrics, Budapeste, v. 67, n. 1, p. $87-105,2006$.

LI, Y.; RADICCHI, F.; CASTELLANO, C.; RUIZ-CASTILHO, J. Quantitative evaluation of altenative field normalization procedures. In: INTERNATIONAL SOCIETY OF SCIENTOMETRICS AND INFORMETRICS CONFERENCE, 14., 2013, Viena.Proceedings ... Viena: ISSI, 2013. p. 1431-1441.

MOED, H.F. New developments in the use of citation analysis in research evaluation. Scientometrics, Budapeste, v. 57, p. 13-18, 2009.

Narin, F.; Olivastro, D.; Stevens, K. S. Bibliometric theory. practice and problem.Evaluation Review, Thousand Oaks (EUA), v. 18, p. 65-76, 1994.

OKUBO, Y.Bibliometric indicators and analysis of research systems: methods and examples. Paris: OECD, 1997

Oliveira, E. F. T.; Grácio, M. C. C. A produção científica em organização e representação do conhecimento no Brasil: uma análise bibliométrica do 
GT-2 da Ancib.In: ENCONTRO NACIONAL DE PESQUISA DA ANCIBENANCIB, 10., 2009 João Pessoa. Anais..... João Pessoa: ANCIB, 2009.

OLIVEIRA, E.F.T.; GRÁCIO, M.C.C. indicadores bibliométricos em ciência da informação: análise dos pesquisadores mais produtivos no tema estudos métricos na base scopus. Perspectivas em Ciência da Informação, Belo Horizonte, v. 16, p. $16-28,2011$.

Persson, O.; Glänzel, W.; Danell, R. Inflationary bibliometric values:The role of scientific collaboration and the need for relative indicators in evaluative studies. Scientometrics, Budapeste, v. 60, n.3, p. 421-432, 2004.

SCImago SJR - SCImago Journal \& Country Rank. 2012. Available from:www.SCImagojr.com, 2012. Access: Dec. 18th 2012.

SPINAK, E. Indicadores cienciométricos. Ciência da Informação, Brasília, DF, v. 27, n. 2, p.141-148, 1998.

Vieira, E. S.; Gomes, J. A. N. F. A research impact indicator for institutions. Journal of Informetrics, Amsterdam, v. 4, n. 4, p. 581-590, 2010.

ZANOTTO. E.D. Scientific and technological development in Brazil. The widening gap. Scientometrics, Budapeste, v. 55, n. 3, p. 411-419, 2002. 